OPEN ACCESS

Edited by:

Marina De Bernard,

University of Padua, Italy

Reviewed by:

Lucia Galli,

CONICET La Plata \& Universidad

Nacional de La Plata, Argentina

Caitlin S. L. Parello,

Biomodels, USA

${ }^{*}$ Correspondence:

Yaxian Yan

yanyaxian@sjtu.edu.cn

Jianhe Sun

sunjhe@sjtu.edu.cn

Specialty section: This article was submitted to

Microbial Immunology,

a section of the journal

Frontiers in Microbiology

Received: 17 January 2017 Accepted: 30 March 2017

Published: 18 April 2017

Citation:

Fu Q, Li S, Wang Z, Shan W, Ma J, Cheng $Y$, Wang $H$, Yan $Y$ and Sun $J$ (2017) H-NS Mutation-Mediated CRISPR-Cas Activation Inhibits Phage Release and Toxin Production of Escherichia coli Stx2 Phage Lysogen. Front. Microbiol. 8:652. doi: 10.3389/fmicb.2017.00652

\section{H-NS Mutation-Mediated CRISPR-Cas Activation Inhibits Phage Release and Toxin Production of Escherichia coli Stx2 Phage Lysogen}

\author{
Qiang Fu, Shiyu Li, Zhaofei Wang, Wenya Shan, Jingjiao Ma, Yuqiang Cheng, \\ Hengan Wang, Yaxian Yan * and Jianhe Sun *
}

Shanghai Key Laboratory of Veterinary Biotechnology, Key Laboratory of Urban Agriculture (South), Ministry of Agriculture, School of Agriculture and Biology, Shanghai Jiao Tong University, Shanghai, China

Shiga toxin-converting bacteriophages (Stx phages) carry the stx gene and convert nonpathogenic bacterial strains into Shiga toxin-producing bacteria. There is limited understanding of the effect that an Escherichia coli (E. coli) clustered regularly interspaced short palindromic repeats (CRISPR)-Cas adaptive immune system has on Stx phage lysogen. We investigated heat-stable nucleoid-structuring (H-NS) mutation-mediated CRISPR-Cas activation and its effect on $E$. coli Stx2 phage lysogen. The $\Delta h n s$ mutant (MG1655 $\Delta h n s$ ) of the E. coli K-12 strain MG1655 was obtained. The $\Delta h n s$ mutant lysogen that was generated after Stx phage lysogenic infection had a repressed growth status and showed subdued group behavior, including biofilm formation and swarming motility, in comparison to the wild-type strain. The de-repression effect of the H-NS mutation on CRISPR-Cas activity was then verified. The results showed that cas gene expression was upregulated and the transformation efficiency of the wild-type CRISPR plasmids was decreased, which may indicate activation of the CRISPR-Cas system. Furthermore, the function of CRISPR-Cas on Stx2 phage lysogen was investigated by activating the CRISPR-Cas system, which contains an insertion of the protospacer regions of the Stx2 phage Min27. The phage release and toxin production of four lysogens harboring the engineered CRISPRs were investigated. Notably, in the supernatant of the $\Delta h n s$ mutant lysogen harboring the Min27 spacer, both the progeny phage release and the toxin production were inhibited after mitomycin $\mathrm{C}$ induction. These observations demonstrate that the H-NS mutation-activated CRISPR-Cas system plays a role in modifying the effects of the Stx2 phage lysogen. Our findings indicated that $\mathrm{H}-\mathrm{NS}$ mutation-mediated CRISPR-Cas activation in E. coli protects bacteria against Stx2 phage lysogeny by inhibiting the phage release and toxin production of the lysogen.

Keywords: Escherichia coli, H-NS, CRISPR-Cas, Stx2 phage, Shiga toxin 


\section{INTRODUCTION}

Shiga toxin (Stx)-producing Escherichia coli (STEC), especially the O157:H7 strains, are the major causes of hemorrhagic colitis and hemolytic-uremic syndrome (HUS) (Allison et al., 2003). Although some bacteriophage infections are lytic, temperate and lysogenic bacteriophages often integrate into the bacterial genome as a prophage causing a chronic infection of the host bacterium, known as lysogenic conversion (Zegans et al., 2009). It is known that Stx phage lysogenization of STEC promotes its pathogenicity and contributes to its viability in environmental conditions (Croxen et al., 2013). Stx2 production is reported to be associated with more severe diseases than strains that produce either Stx1 or a combination of Stx1 and Stx2 (Kleanthous et al., 1990; Pacheco and Sperandio, 2012; Kruger and Lucchesi, 2015). Clinically, the use of antibiotics to treat STEC infections has become controversial due to the stimulation of the lytic cycle and the concomitant toxin release through the bacterial SOS response (Croxen et al., 2013). As described previously, when phages infect bacterial cells, they must resist a range of antiviral mechanisms in order to thrive in most environments (Labrie et al., 2010). As an important bacteriophage resistance mechanism, a clustered regularly interspaced short palindromic repeats (CRISPR)-Cas system plays a vital role in preventing phage infection and multiplication.

The CRISPR-Cas system is a sophisticated adaptive immune system in prokaryotes that consists of short DNA repeats separated by $26-72$ bp sequences, called spacers, which are derived from viruses and other foreign DNA (van der Oost et al., 2009; Yin et al., 2013). In addition to the CRISPR-Cas system's role in adaptive immunity, its function in regulating the behavior of bacteria has also been widely reported (Westra et al., 2014; Fu et al., 2015). Moreover, due to the high polymorphisms of CRISPR arrays, CRISPR sequences have been successfully used to classify a number of bacteria in investigations of infectious disease outbreaks (Liu et al., 2011; Louwen et al., 2014; Fu et al., 2017). Complementarity between mature CRISPR RNAs (crRNAs) and the sequences found within phages or plasmids activate Cas proteins, which target and cleave the complementary sequence, leading to the inhibition of their infection or replication (Garneau et al., 2010; Westra et al., 2012).

In strains of Escherichia coli (E. coli), such as E. coli K-12 MG1655, the type I-E CRISPR-Cas immune system consists of two major CRISPR loci and eight cas genes: cas1, cas2, cas3, and cas $A B C D E$. However, the activity of the E. coli CRISPR-Cas system is repressed by the heat-stable nucleoid structuring (H-NS) protein, a global transcriptional repressor. As one of the most abundant proteins in the E. coli nucleoid, H-NS is also widely distributed within other gram-negative bacteria (Bloch et al., 2003; Dorman, 2004, 2014). Thus, relieving H-NS-mediated repression of cas gene transcription may be a primary prerequisite for the CRISPR-Cas system's ability to resist phage lysogenization, lysogens, and prophage induction (Pul et al., 2010; Majsec et al., 2016). Since the Stx phage plays an important role in the dissemination of Shiga toxin genes and the emergence of new STEC strains, how the CRISPR-Cas system will perform against a Stx prophage integrated into a bacterial genome must be further elucidated. In this study, the E. coli $\mathrm{O} 157: \mathrm{H} 7$ strain Min27 was used; this pathogenic strain, which was isolated in the field, is capable of producing Shiga toxin after induction by either mitomycin $\mathrm{C}$ or antibiotics, such as norfloxacin and gentamicin. The activated CRISPR system's effect on the phage release, toxin production, and other biological characters of phage lysogen were determined. Since the impact of the CRISPR-Cas system on Stx2 phage lysogen is uncertain, in this study a $\Delta h n s$ mutant of $E$. coli K-12 was generated to activate the system. Based on the general feature of spacer-protospacer matching of an E. coli CRISPR-Cas system, a Stx2 phage Min27 single lysogen of the $\Delta h n s$ mutant and different lysogens that harbor the engineered CRISPRs were generated. The Stx 2 phage resistant function of an H-NS-mediated E. coli type I-E CRISPRCas system was investigated by testing the lysogenization efficiency, phage release, and Shiga toxin production. The findings suggest that the CRISPR-Cas immune system impacts the biological characteristics of Stx phage lysogen in E. coli.

\section{MATERIALS AND METHODS}

\section{Bacterial Strains, Bacteriophages, and Media}

The bacterial strains, bacteriophages, and plasmids used in this study are listed in Table 1. E. coli K-12 strain MG1655 was used in this study. The E. coli K-12 derivative strain, MC1061, was used as a nontoxigenic host for Stx phage infections and propagation (Allison et al., 2003). The E. coli strains were routinely cultured in Luria-Bertani (LB) broth (Oxoid, Basingstoke, U.K.) or LB agar $(1.5 \%)$ at $37^{\circ} \mathrm{C}$. The growth media were supplemented with the following antibiotics at the indicated concentrations: ampicillin (Amp), $100 \mu \mathrm{g} / \mathrm{ml}$; chloramphenicol (Cm), $34 \mu \mathrm{g} / \mathrm{ml}$. The Stx2 phage Min27 was obtained from E. coli O157:H7 strain Min27, which was isolated from the feces of a piglet with diarrhea at a swine farm in Shanghai, China (Su et al., 2010). Recombinant mutant phage Min27( $\Delta$ stx::cat) was constructed using the lambda Red recombinase method with Stx $2 \mathrm{AB}$ deleted, but carrying a chloramphenicol resistance cassette. This mutant phage was used in the latter experiment of lysogenic infection efficiency for colony-forming unit (CFU) counting of the lysogen on the $\mathrm{Cm}^{\mathrm{R}}$ plate. The related primers used for mutation and verification of the recombinant phage are listed in Table 2.

\section{Plasmid Construction}

The plasmids were constructed using standard molecular biology techniques. Standard digestion of the polymerase chain reaction (PCR) products and the vector by restriction enzymes was conducted according to the manufacturer's instructions. The CRISPR1 and CRISPR2 loci of MG1655 were amplified using specified primers, and then they were inserted into the $B a m H$ I-Eco R I-cleaved pGEX-6p-1 vector to generate the foreign plasmids pCRISPR1 and pCRISPR2, respectively. The 
TABLE 1 | E. coli K-12 bacterial strains, bacteriophages, and plasmids used in this study.

\begin{tabular}{|c|c|c|}
\hline Strains and plasmids & Genotype/Relevant characteristics & Source \\
\hline \multicolumn{3}{|l|}{ BACTERIA } \\
\hline MG1655 & Wild-type E. coli K-12 & This study \\
\hline MG1655 $\Delta h n s$ & $\mathrm{H}-\mathrm{NS}$ deletion & This study \\
\hline SL & MG1655ФMin27, Wild-type Min27 phage single lysogen & This study \\
\hline SL $\Delta h n s$ & MG1655 $\Delta h n s \Phi M i n 27, h n s$ deletion then lysogenic infected by single wild-type Min27 phage & This study \\
\hline MC1061 & Indicator strain (E. coli K-12) & This study \\
\hline FQ1 & MG1655 anti-control spacer, MG1655 harboring the engineered CRISPR plasmid with control spacers; Amp ${ }^{R}$ & This study \\
\hline FQ2 & $\begin{array}{l}\text { MG1655 anti-Min27 spacer, MG1655 harboring the engineered CRISPR plasmid with spacers derived from the } \\
\text { phage Min27 genome; AmpR }\end{array}$ & This study \\
\hline FQ3 & $\begin{array}{l}\text { MG1655 } 4 h n s \text { anti-control spacer, MG1655 with hns deletion harboring the engineered CRISPR plasmid with } \\
\text { control spacers; Amp }\end{array}$ & This study \\
\hline FQ4 & $\begin{array}{l}\text { MG1655 } \Delta h n \text { s anti-Min27 spacer, MG1655 with } h n s \text { deletion harboring the engineered CRISPR plasmid with } \\
\text { spacers derived from the phage Min27 genome; } \mathrm{Amp}^{R}\end{array}$ & This study \\
\hline FQ5 & $\begin{array}{l}\text { SL anti-control spacer, Wild-type Min27 phage single lysogen harboring the engineered CRISPR plasmid with } \\
\text { control spacers; Amp }\end{array}$ & This study \\
\hline FQ6 & $\begin{array}{l}\text { SL anti-Min27 spacer, Wild-type Min27 phage single lysogen harboring the engineered CRISPR plasmid with } \\
\text { spacers derived from the phage Min27 genome; } \mathrm{Amp}^{R}\end{array}$ & This study \\
\hline FQ7 & $\begin{array}{l}\text { SL } \Delta h n s \text { anti-control spacer, Wild-type Min27 phage single lysogen with } h n s \text { deletion harboring the engineered } \\
\text { CRISPR plasmid with control spacers; } \mathrm{Amp}^{R}\end{array}$ & This study \\
\hline FQ8 & $\begin{array}{l}\text { SL } \Delta h n s \text { anti-Min27 spacer, Wild-type Min27 phage single lysogen with hns deletion harboring the engineered } \\
\text { CRISPR plasmid with spacers derived from the phage Min27 genome; Amp }\end{array}$ & This study \\
\hline \multicolumn{3}{|l|}{ BACTERIOPHAGES } \\
\hline$\Phi M i n 27$ & Wild-type Stx2 phage Min27; isolated from O157:H7 strain Min27 & $\begin{array}{l}\text { Su et al., } \\
2010\end{array}$ \\
\hline$\Phi$ Min27( $\Delta$ stx::cat) & Recombinational phage Min27 with Stx deletion, but carrying chloramphenicol resistance; $\mathrm{Cm}^{\mathrm{R}}$ & This study \\
\hline \multicolumn{3}{|l|}{ PLASMIDS } \\
\hline pKD46 & Amp ${ }^{R}, \lambda$ Red recombinase expression & $\begin{array}{l}\text { Datsenko and } \\
\text { Wanner, } 2000\end{array}$ \\
\hline pKD3 & Chloramhenicol resistance cassette & $\begin{array}{l}\text { Datsenko and } \\
\text { Wanner, } 2000\end{array}$ \\
\hline pCP20 & Temperature sensitive replication, thermal induction of FLP recombinase synthesis; $\mathrm{Amp}^{R}$ and $\mathrm{Cm}^{\mathrm{R}}$; & $\begin{array}{l}\text { Datsenko and } \\
\text { Wanner, } 2000\end{array}$ \\
\hline pGEX-6p-1 & Empty vector, $\mathrm{Amp}^{\mathrm{R}}$ & This study \\
\hline pGEX-6p-1-CRISPR1 & pGEX-6p-1 cloned with K-12 CRISPR1 loci & This study \\
\hline pGEX-6p-1-CRISPR2 & pGEX-6p-1 cloned with K-12 CRISPR2 loci & This study \\
\hline pGEX-6p-1-anti-control spacer & Engineered CRISPR plasmid with control spacers & This study \\
\hline pGEX-6p-1-anti-Min27 spacer & Engineered CRISPR plasmid with spacers derived from the phage Min27 genome & This study \\
\hline
\end{tabular}

$\mathrm{Cm}^{R}$ and $\mathrm{Amp}^{R}$ represent chloramphenicol and ampicillin resistance, respectively. Strains harboring engineered CRISPR plasmid were referred to by the identifiers (FQ1-8).

construction of the plasmids pCRISPR1, pCRISPR2, the p-anticontrol spacer, and the p-anti-Min27 spacer, are described in the Supplementary Material.

\section{$\Delta h n s$ Mutant Construction}

The mutant with an in-frame deletion in the $h n s$ gene was generated using the lambda Red system, as described previously (Datsenko and Wanner, 2000; Wang et al., 2011). The hns gene was replaced with a chloramphenicol resistance cassette, which was amplified from plasmid pKD3 using PCR with hns deletion primers (Table 2). The $5^{\prime}$ regions of the primers (50 bp) were homologous to the corresponding flanking region of the hns. The PCR products were then transformed into MG1655 containing the lambda Red recombinase expression plasmid pKD46 by electroporation. After electroporation, the samples were incubated at $37^{\circ} \mathrm{C}$ for $1 \mathrm{~h}$ in Super Optimal Broth with Catabolite repression (SOC) and plated on LB agar with chloramphenicol to select the $\Delta h n s$ mutants. The mutants were confirmed by PCR and sequenced using $\Delta h n s$ mutant verification primers. The chloramphenicol resistance cassette was cured by transforming the $\mathrm{pCP} 20$ plasmid into the mutant and selecting for a chloramphenicol sensitive mutant strain, which was finally designated as MG1655 $h$ hns.

\section{Generation of the Phage Lysogens and Measurements of the Phage Titer}

To generate the Stx phage lysogens, $100 \mu$ l of filtered lytic supernatants containing phage (prepared as described above) was mixed with $100 \mu \mathrm{l}$ of $E$. coli K-12 MG1655 cells from an overnight LB-grown culture, and the MG1655 and phage mixture 
TABLE 2 | Oligonucleotide primers used in this study.

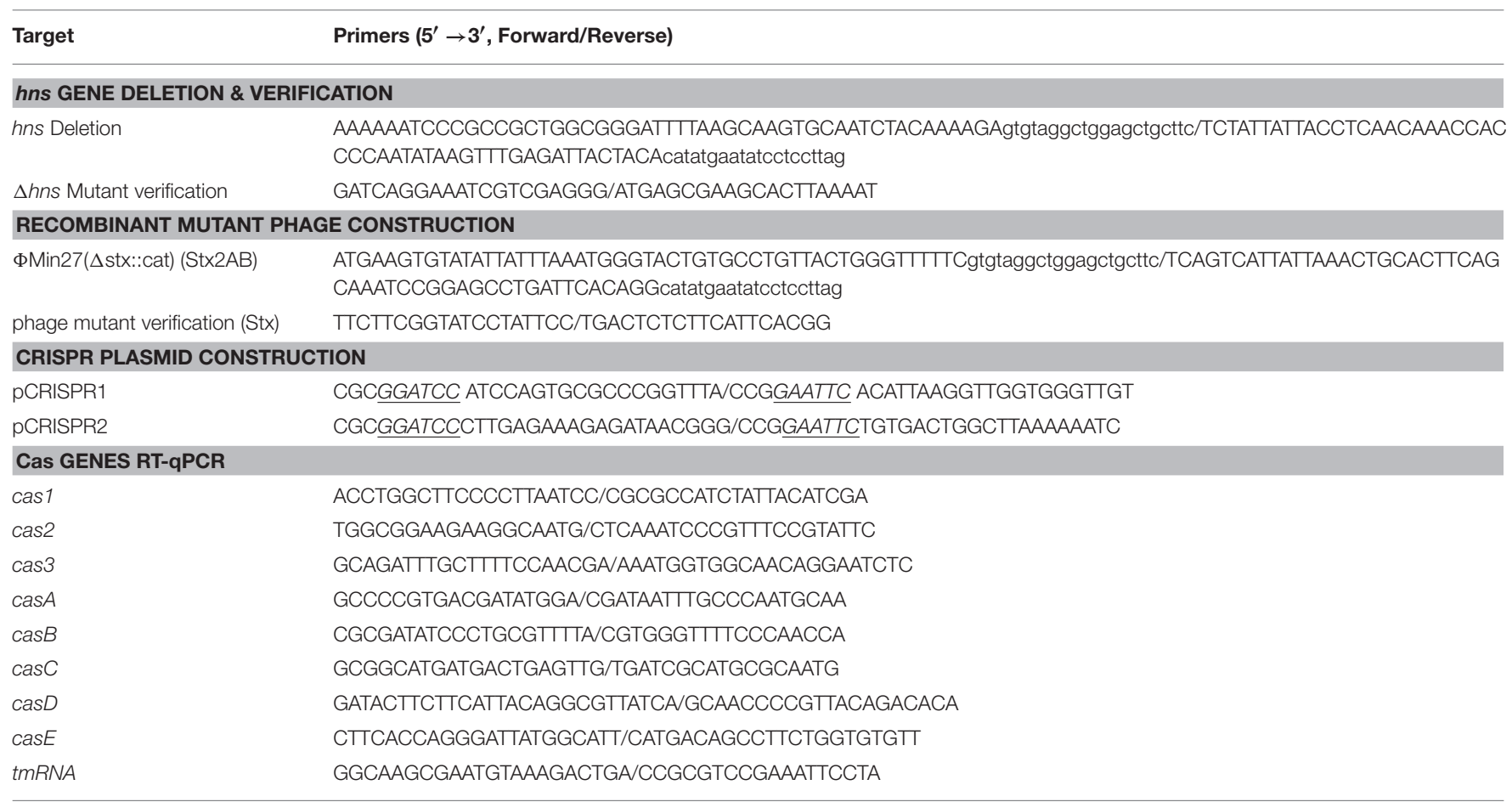

Underline and italic texts indicate restriction enzyme sites. GGATCC is the recognition site for BamH I and GAATTC is the recognition site for ECo $R$ I.

was incubated overnight at $37^{\circ} \mathrm{C}$ in $\mathrm{LB}$ medium. These bacteriaphages mixtures were then struck on plates to obtain individual colonies. The individual colonies were inoculated into liquid LB medium, grown overnight, and then assayed for the presence of phage. The lytic supernatant-containing phage was serially diluted and incubated with indicator strain MC1061 (grown in $\mathrm{LB}$ until $\mathrm{OD}_{600}$ reached 0.4 ) plus $0.01 \mathrm{M} \mathrm{CaCl}_{2}$ at $37^{\circ} \mathrm{C}$ for 15 $\mathrm{min}$. The cells were mixed with top agar $(5 \mathrm{ml})$ and poured onto $\mathrm{LB}$ agar plates. After incubation at $37^{\circ} \mathrm{C}$ for $8 \mathrm{~h}$, the phage titer expressed as plaque-forming units $(\mathrm{PFU}) / \mathrm{ml}$ was calculated by counting the plaques on the plate.

\section{Biofilm Formation Assay}

Biofilm formation assays were performed using a previously described, 96-well plate method with some modifications (Schembri and Klemm, 2001; Skyberg et al., 2007). In brief, single lysogens were grown to stationary phase in $\mathrm{LB}$ at $37^{\circ} \mathrm{C}$ and then diluted 1:100 in LB supplemented with $5 \mathrm{~g} / \mathrm{L}$ glucose. Aliquots of $200 \mu \mathrm{l}$ for each dilution were dispensed per well into a microtiter plate. The negative-control wells contained uninoculated medium. The plates were cultured aerobically at $37^{\circ} \mathrm{C}$ without shaking for $24 \mathrm{~h}$. The medium of the plates was then poured off, and the plates were triple-washed with sterile phosphate-buffered saline (PBS). The microplates were then stained with $200 \mu \mathrm{l}$ of $1 \%(\mathrm{w} / \mathrm{v})$ crystal violet for 30 min, washed three times with PBS to remove the unbound crystal violet dye, and air dried for $1 \mathrm{~h}$. After drying, the adherent cells were re-solubilized with $200 \mu \mathrm{l}$ of $30 \%$ acetic acid. The absorbance was measured at $550 \mathrm{~nm}$ in a microplate
(ELISA) reader (BioTek, ELx800). All tests were carried out three times independently, and the results were analyzed statistically.

\section{Swarming Mobility Assay}

Swarming assays were performed as reported previously (Toutain et al., 2005; Su et al., 2010). The swarming motility of the single lysogens was tested by inoculating $5 \mu \mathrm{l}$ of an overnight, LBgrown culture onto a swarming plate followed by incubation at $37^{\circ} \mathrm{C}$ for $48 \mathrm{~h}$. A semisolid medium containing $0.5 \%$ agar was supplemented with $0.5 \% \mathrm{D}$-(+)-glucose. The swarming motility plates were prepared and used at the same day, after solidification of agar, they were dried for 5 min under sterile air and then inoculated.

\section{Quantitative Real-Time Reverse Transcription-PCR}

The bacteria were grown to the logarithmic phase, and RNA was isolated using the E.Z.N.A. bacterial RNA isolation kit (Omega, Beijing, China) according to the manufacturer's instructions. Contaminated DNA was removed from the samples with RNase-free DNase I (Thermo Scientific, Waltham, MA, USA), and cDNA synthesis was performed using the Reverse Transcription System (Promega, Madison, WI, USA) according to the manufacturer's instructions. Quantitative real-time reverse transcription-PCR (RT-qPCR) was performed to determine the transcription levels of the cas genes using SYBR Green MasterMix (DBI Bioscience, Ludwigshafen, Germany) and gene-specific primers (Table 2), and the data were normalized with the 
housekeeping gene tmRNA transcript. The relative fold change was calculated using the threshold cycle $\left(2^{-\Delta \Delta C T}\right)$ method.

\section{Transformation Efficiency Assay}

The E. coli MG1655 and MG1655 $4 h n s$ cultures were incubated at $37^{\circ} \mathrm{C}$ until an $\mathrm{OD}_{600}$ of $0.5-0.6$ was reached. The bacteria were then centrifuged at $4,000 \times \mathrm{g}$ at $4^{\circ} \mathrm{C}$, the supernatants were disposed, and the bacteria were resuspended in $1 \mathrm{ml}$ icecold $10 \%$ glycerol, and then transferred to a $1.5 \mathrm{ml}$ tube. After additional washing steps, the cells were suspended in $200 \mu$ licecold $10 \%$ glycerol. The bacterial cells $(50 \mu \mathrm{L})$ were then mixed in an ice-cold $0.2 \mathrm{~mm}$ electroporation cuvette (Bio-Rad) with 2 $\mu \mathrm{g}$ of the pVEC, pCRISPR1, or pCRISPR2 plasmids. The mixture was pulsed in a Bio-Rad MicroPulser at $200 \Omega, 25 \mu \mathrm{F}$, and $1.8 \mathrm{kV}$. Immediately after the pulse, $1 \mathrm{ml}$ of SOC broth was added, and the cells were aerated for $2 \mathrm{~h}$ at $37^{\circ} \mathrm{C}$. Various dilutions of the reaction were plated on $\mathrm{LB}$ agar plates supplemented with 100 $\mu \mathrm{g} / \mathrm{ml}$ Ampcillin. The plates were incubated overnight at $37^{\circ} \mathrm{C}$. The colonies emerging on the selection plates were counted, and the CFU number per microgram plasmid was calculated, accordingly.

\section{Lytic and Lysogenic Infection Efficiency Assay}

The E. coli MG1655 and MG1655 $4 h n s$ cultures harboring the engineered CRISPRs (containing either the control spacer or the Min27 spacer) (FQ1, FQ2, FQ3, and FQ4 in Table 1) were incubated at $37^{\circ} \mathrm{C}$ until an $\mathrm{OD}_{600}$ of $0.5-0.6$ was reached. All the strains were normalized to $\mathrm{OD}_{600}=0.6$, and the $\mathrm{CFU}$ was also counted and calculated on the Amp ${ }^{\mathrm{R}}$ plate. For lytic infection, aliquot supernatants containing infectious Min27 phage particle was added to $5 \mathrm{ml}$ of the normalized cultures and then cultured aerobically at $37^{\circ} \mathrm{C}$ with shaking for $12 \mathrm{~h}$, respectively. The cultures were then centrifuged at $4,000 \times \mathrm{g}$ for $5 \mathrm{~min}$, and the supernatants were used to determine the phage titer via PFU assay. The efficiency of the plating (EOP) (EOP = phage titer on test bacterium/phage titer on indicator bacterium MC1061) was also measured. For the lysogenic infection, the recombinant mutant phage Min27( $\Delta$ stx::cat), which carries a chloramphenicol resistance cassette, was used. A total of $100 \mu \mathrm{l}$ of the filtered phage Min27( $\Delta$ stx::cat) was mixed with $100 \mu$ l of the E. coli MG1655 and MG1655 $\Delta h n s$ cultures harboring the engineered CRISPRs, and the mixtures were incubated overnight at $37^{\circ} \mathrm{C}$. These bacterium-phage mixtures were then struck on $A m p^{R}$ (lysogens and non-lysogens cells) or $\mathrm{Amp}^{\mathrm{R}}$ plus $\mathrm{Cm}^{\mathrm{R}}$ (lysogens) plates to obtain individual colonies and to calculate the CFUs.

\section{Phage Release of the Lysogens}

The lysogens harboring the engineered CRISPR (FQ5, FQ6, FQ7, and FQ8) and the control strains were induced to release the phage by exposing the mid-exponential-phase cultures (all normalized to $\left.\mathrm{OD}_{600}=0.6\right)$ of the representative ampicillinresistant colonies to mitomycin C $(0.5 \mu \mathrm{g} / \mathrm{ml})$ (Sigma) (4) for 12 h. The progeny phage titer was determined using the PFU assay described above. Furthermore, the lysogen survival rate after mitomycin $\mathrm{C}$ induction was also calculated using CFU counting.

\section{Vero Cell Viability Assay}

The Vero cells were plated at $10^{4} /$ well on 96 -well plates in Dulbecco's Modified Eagle Medium (DMEM) containing 10\% fetal bovine serum, and then incubated overnight at $37^{\circ} \mathrm{C}$ under $5 \% \mathrm{CO}_{2}$. Four lysogens FQ5, FQ6, FQ7, and FQ8 were induced to release the phage and Shiga toxin by exposing the midexponential-phase cultures (all normalized to $\mathrm{OD}_{600}=0.6$ ) of the representative ampicillin-resistant colonies to mitomycin $\mathrm{C}$ (0.5 $\mu \mathrm{g} / \mathrm{ml})$ (Sigma) (4) for $12 \mathrm{~h}$. Serial 10-fold dilutions of the filtered $(0.22 \mu \mathrm{m}$ filter $)$ supernatants in DMEM medium were prepared. Dilutions $\left(10^{-1}-10^{-4}\right)$ were added to the cell monolayer $(100 \mu \mathrm{l} /$ well $)$ and incubated for $48 \mathrm{~h}$ at $37^{\circ} \mathrm{C}$ under $5 \% \mathrm{CO}_{2}$. The viability of the Vero cells was determined via crystal violet staining. All data was represented as the average of triplicate assays. A lysogen without cytotoxic effect, MG1655ФMin27( $\Delta$ stx::cat), was used as a negative control.

\section{Western Blot Analysis}

A total of $10 \mathrm{ml}$ the lysogens harboring the engineered CRISPR (FQ5, FQ6, FQ7, and FQ8) were induced to release the phage and Shiga toxin by exposing the cultures (all normalized to $\left.\mathrm{OD}_{600}=0.6\right)$ of the representative ampicillin-resistant colonies to mitomycin C $(0.5 \mu \mathrm{g} / \mathrm{ml})$ for $12 \mathrm{~h}$. The filtered supernatants were collected and then subjected to saturated ammonium sulfate precipitation. The mixture of the supernatants and the saturated ammonium sulfate (1:1) was gently shaken at $4^{\circ} \mathrm{C}$ for $6 \mathrm{~h}$. The resulting precipitation was dissolved in $1 \mathrm{ml}$ PBS after centrifugation at $10,000 \times \mathrm{g}$ for $10 \mathrm{~min}$. The prepared samples were subjected to sodium dodecyl sulfate (SDS)-polyacrylamide gel electrophoresis (PAGE), and then examined via Western blot analysis using the Stx2 monoclonal antibody (MAb) 11E10 (Santa Cruz, Dallas, USA), as previously described (Smith et al., 2009).

\section{Statistical Analyses}

The reported differences were analyzed using either Student's $t$-test (Figure 2B) or one-way analysis of variance (ANOVA) (Figure 6A). The analyses were conducted using GraphPad Prism 5 software. A $P<0.05$ was considered to be statistically significant.

\section{RESULTS}

\section{H-NS Deletion Mediates E. coli K-12 CRISPR-Cas Activation}

It has been reported that, under laboratory conditions, transcription of the Cascade genes in the E. coli CRISPR-Cas system is repressed by H-NS (Edgar and Qimron, 2010; Pul et al., 2010; Majsec et al., 2016). In order to investigate the interactions between CRISPR-Cas and phage lysogen, a mutant with $h n s$ gene in-frame deletion was generated (Figure 1A). To study the effect of $h n s$ deletion on CRISPR-Cas activation and cas gene expression, the transcription levels of the wild-type MG1655 and the $\Delta h n s$ strain cas genes in the mid-exponential growth phase were examined via real-time quantitative polymerase chain reaction (RT-qPCR). The $\Delta h n s$ strain revealed a significant upregulation of the transcriptions of cas $A B C D E$, cas 1 , cas 2 , and cas 3 (average 5-fold difference) than the wild-type strain 

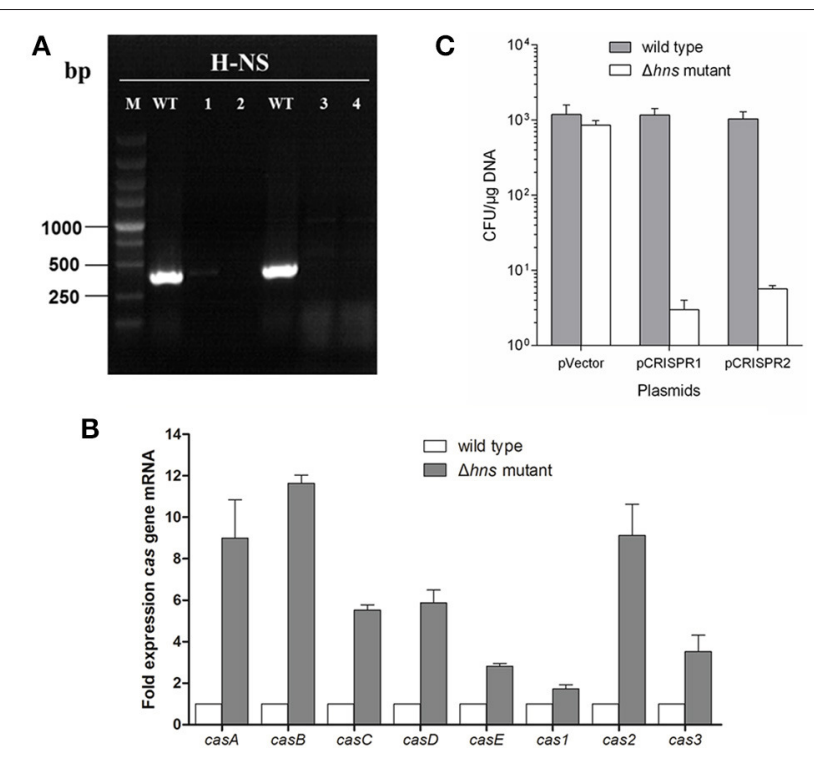

FIGURE 1 | H-NS deletion mediates E. coli K-12 CRISPR-Cas activation. (A) The mutant with an in-frame deletion in the $h n s$ gene was generated and verified by PCR. Line M indicates a DL5000 DNA marker; Line WT indicates the wild-type strain. Lines 1, 2, 3, and 4 indicate four independent mutant colonies. The results show that the $h n s$ genes of the four mutants were successfully deleted. Line 2 was chosen as the $\Delta h n s$ mutant for future experiments. (B) Cas gene transcription levels of the wild-type (MG1655) and $\Delta h n s$ mutant (MG1655 $h$ hns) strains in the mid-exponential growth phase were examined using the RT-qPCR method. (C) Transformation efficiency of the foreign plasmids encoding the homologous spacer with the $E$. coli CRISPR loci was determined. Plasmids harboring two CRISPR loci, CRISPR1 and CRISPR2, as well as an empty vector, were transformed into the $\Delta h n s$ and wild-type strains, and the transformation efficiencies were calculated, respectively.

(Figure 1B). These results are consistent with the findings reported in previous studies (Westra et al., 2010), indicating that cas gene transcription is closely associated with H-NS.

It has been demonstrated that the CRISPR-Cas system provided protection against invading mobile genetic elements (MGEs), such as plasmids and phages (Barrangou and Marraffini, 2014; Barrangou, 2015). To further verify that H-NS deletion mediates CRISPR-Cas activation, the transformation efficiency of the foreign plasmids that carry the homologous spacer with E. coli CRISPR loci was determined. Plasmids harboring two CRISPR loci, known as CRISPR1 and CRISPR2, as well as an empty vector, were transformed into the $\Delta h n s$ mutant and wild-type strains, and the transformation efficiencies were calculated, respectively. The plasmid containing the CRISPR1 sequences (with the same protospacer against the CRISPR1 loci) transformed into the $\Delta h n s$ mutant with an efficiency that was approximately $0.35 \%$ of the efficiency of the empty vector, but no obvious difference in transformation efficiency was observed in the wild-type strain (Figure 1C). Similarly, transformation of the plasmids containing the CRISPR2 protospacer into MG1655 $4 h n s$ was reduced by around $90 \%$ in comparison to the empty vector. In contrast, no reduction in the transformation efficiency was observed for the plasmids when they were introduced into the wild-type strain (Figure 1C). These results clearly demonstrate that the proposed E. coli CRISPR-Cas system is active against foreign plasmids after relieving the $h n s$ repression.

\section{H-NS Regulates the Group Behavior of the E. coli Stx2 Phage Lysogen}

To access the possible role of H-NS mutation-mediated CRISPRCas immunity in regulating these bacterial group behaviors, we evaluated the biofilm formation and swarming motility ability of the Stx2 phage lysogenic wild-type and $\Delta h n s$ mutant strains. The results showed that the swarming mobility of the $\Delta h n s$ mutant lysogen was extremely inhibited in comparison to the wildtype Min27 phage lysogen (Figure 2A). In bacteria lysogenized with bacteriophage Min27, the $\Delta$ hns mutant lysogen showed decreased biofilm formation ability in comparison to the wildtype Min27 phage lysogen (Figure 2B). Biofilm formation is another type of bacterial group behavior. The results suggest that lysogeny by Stx phage Min27 interferes with at least two E. coli group behaviors. It was also observed that lysogenic infection of Pseudomonas aeruginosa PA14 with bacteriophage DMS3 inhibits bacterial group behaviors, such as biofilm formation and swarming motility, which were later demonstrated to be associated with the CRISPPR-Cas system function (Zegans et al., 2009). These observations indicate that the activated E. coli CRISPR-Cas system may also be involved in inhibiting the group behavior of the Stx2 phage lysogen.

\section{Activated CRISPR-Cas Inhibits Phage Replication}

To evaluate the possible function of CRISPR-Cas-associated immunity in inhibiting the replication of phages in the E. coli K-12 stains, four strains of the wild-type and $\Delta h n s$ mutant harboring the engineered CRISPRs (containing either the control spacer or the Min27 phage-derived spacer) were generated: FQ1, FQ2, FQ3, and FQ4. These were used to detect their ability to hamper phage propagation (Figure 3A). After infecting the strains with the Stx2 phage Min27, progeny phage titer in the hnsdeleted strain harboring the Min27 phage-derived spacer showed a nearly 100 -fold decrease in comparison to the other strains, $12 \mathrm{~h}$ post-infection (Figure 3B). Further evidence showing that H-NS mediates CRISPR-based immunity against the Stx2 phage was obtained using the efficiency of plaquing (EOP) assay. After introducing the Min27-derived spacer CRISPR plasmid into the $\Delta h n s$ mutant (FQ4), it reduced the sensitivity to the Stx2 phage Min27 by 100 -fold, in contrast to the wild-type strain or the strains harboring the control spacer CRISPR plasmid (FQ1, FQ2 and FQ3) (Figure 3C). These data show that the hns mutation containing the Min27 phage-derived spacer plasmid led to a dramatic CRISPR-Cas-mediated inhibition of phage Min27 replication (Figure 3A), and it also contributed to a reduction in the efficiency of plaquing (Figure 3C).

\section{Activated CRISPR-Cas Represses Phage Lysogenic Infection}

To further elucidate the immune mechanism of CRISPRCas against the Stx2 phage, which is mediated by H-NS, 
A

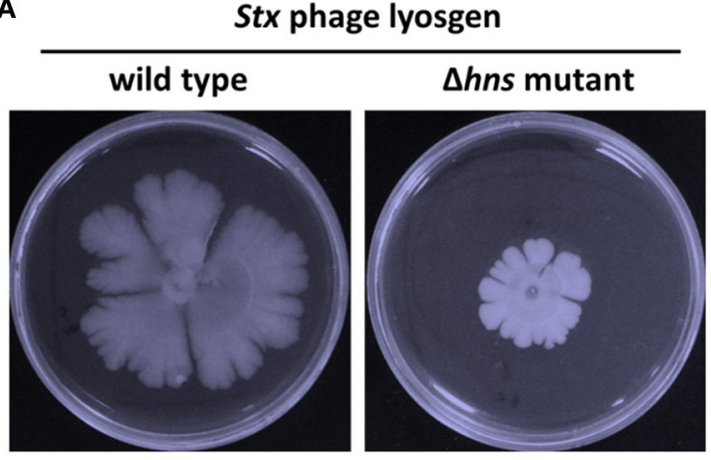

B

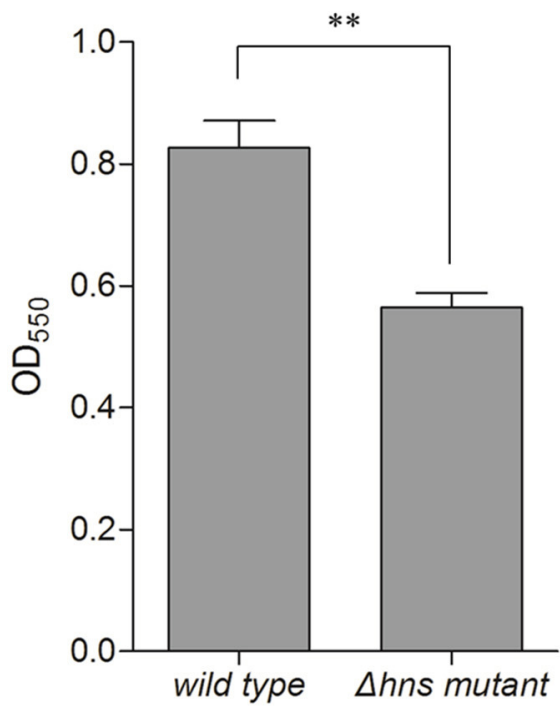

Stx phage lysogen

FIGURE 2 | H-NS regulates the group behavior of the $E$. coli Stx2 phage lysogen. The biofilm formation and swarming motility ability of the Stx2 phage lysogenic wild-type and $\Delta h n s$ mutant strains were assessed. (A) Swarming motility of the single lysogens was tested by inoculating $5 \mu$ l of an overnight, LB-grown culture onto a swarming plate followed by incubation at $37^{\circ} \mathrm{C}$ for $48 \mathrm{~h}$. (B) Biofilm formation was assessed by crystal violent staining of the biofilms grown in microplate wells for $24 \mathrm{~h}$. The data is representative of at least three independent experiments. Student's $t$-tests were used to determine statistically significant differences $(p<0.05) .{ }^{*} p<0.05 ;{ }^{* *} p<0.01$.

the recombinant mutant phage $\operatorname{Min} 27(\Delta$ stx::cat) was used to lysogenically infect the wild-type and $\Delta h n s$ mutant, each of which were harboring the engineered CRISPRs (FQ1, FQ2, FQ3, and FQ4), respectively. Then, the lysogenic infection efficiency of the Stx 2 phage against the above strains was evaluated by counting the CFUs. The stx2 gene from the toxin operon was interrupted by a chloramphenicol resistance cassette (see Materials and Methods), which is convenient for counting the CFUs. After infection, the bacterium-phage mixtures were serialdiluted and then plated onto $\mathrm{Amp}^{\mathrm{R}}$ (lysogenic and non-lysogenic cells) or $\mathrm{Amp}^{\mathrm{R}}$ plus $\mathrm{Cm}^{\mathrm{R}}$ (lysogenic cells) plates to obtain individual colonies and to calculate the $\mathrm{CFU}$ count, respectively.
As shown in Figure 4, there was no significant difference among the CFU counts for the lysogenic and non-lysogenic cells grown on the $A m p^{R}$ plates, which may indicate that the phage had initiated the lysogenic cycle, so that it did not result in a great mass of bacterial lysis. However, for the $\mathrm{Amp}^{\mathrm{R}}$ plus $\mathrm{Cm}^{\mathrm{R}}$ plates, on which the lysogenic cells were screened and counted, the efficiency of the lysogenic infection against FQ4 was greatly reduced $\left(\sim 10^{2}\right.$ times) in comparison to the other three strains (FQ1, FQ2, and FQ3) (Figure 4). These data imply that the activated CRISPR-Cas system in the $\Delta h n s$ E. coli MG1655 strain strongly dampens the lysogenic infection of the Stx2 phage.

\section{Phage Release of the Lysogen Was Targeted by CRISPR-Cas System}

To evaluate the effect of the CRISPR-Cas system on the phage release of the lysogens, mitomycin $\mathrm{C}$ was added to the culture to induce phage release. Then, the titers of the supernatants containing the phages were determined. As shown in Figure 5, the phage titer produced by the Min27 phage lysogen with the CRISPR plasmid carrying the anti-Min27 spacers (FQ8) was approximately 100 -times lower than the $\Delta h n s$ lysogen with the control spacer (FQ7). It was also observed that the phage titer of the wild-type lysogen harboring either Min27 spacer (FQ6) or the control spacer (FQ5) was not remarkably affected. This result indicates that the phage release of the lyosgen harboring the Min27 spacers is targeted by the H-NS-mediated CRISPR activity. Thus, the presence of spacers derived from the phage Min27 is harmful for $\Delta h n s$ mutant lysogen in the phage release after mitomycin C induction.

\section{Shiga Toxin Expression of the Lysogen Was Blocked by the CRISPR-Cas System}

Since the activated CRISPR-Cas system exerts an influence on the phage release of the lysogen, the Shiga toxin expression of the lysogen after induction might also be affected by the CRISPR activity. To verify the effect of CRISPR-Cas, the cytotoxic effect of the supernatants on Vero cells was measured and the Shiga toxin expression was evaluated via Western blot analysis using Stx2 monoclonal antibody (11E10), respectively. To evaluate the potential damage induced by Shiga toxin, the cytotoxicity was evaluated in Vero cells. The Vero cell viability assay results showed that, although the cell death in the $\Delta h n s$ mutant lysogen containing the Min27 spacer (FQ8) was only about $20 \%$, on average, the cell death caused by the culture supernatants from the other three lysogens (FQ5, FQ6, and FQ7) was approximately $40 \%$. In comparison to the other lysogens, the cytotoxic effect of FQ8 was significantly alleviated (Figure 6A). As a control, the supernatants of the recombinant mutant Min27 phage lysogen, MG1655 $\Phi$ Min27( $\Delta$ stx::cat) with no Shiga toxin expression, showed inconspicuous toxicity to the cells. This confirms that the cell death was mainly caused by the Shiga toxin. Likewise, Western blot analysis of the Shiga toxin expression also demonstrated that, with hns deletion, the reduction of Shiga toxin production was greater in the single lysogen harboring the Min27 spacer than it was in the other lysogens, indicating that 
A

Engineered CRISPRs

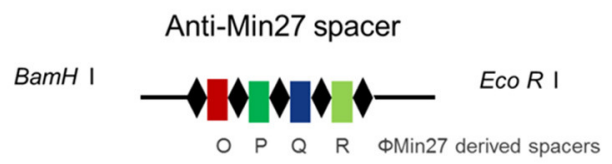

Anti-Control spacer

$\mathrm{BamH}$ I

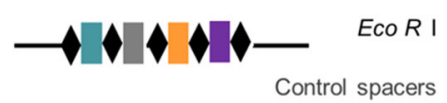

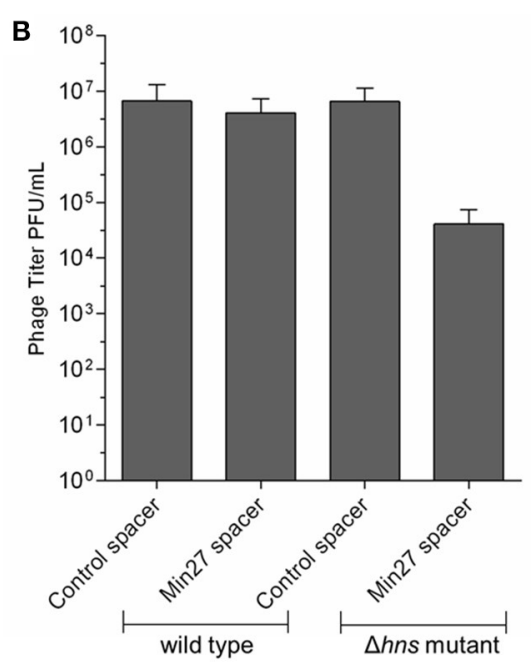

C

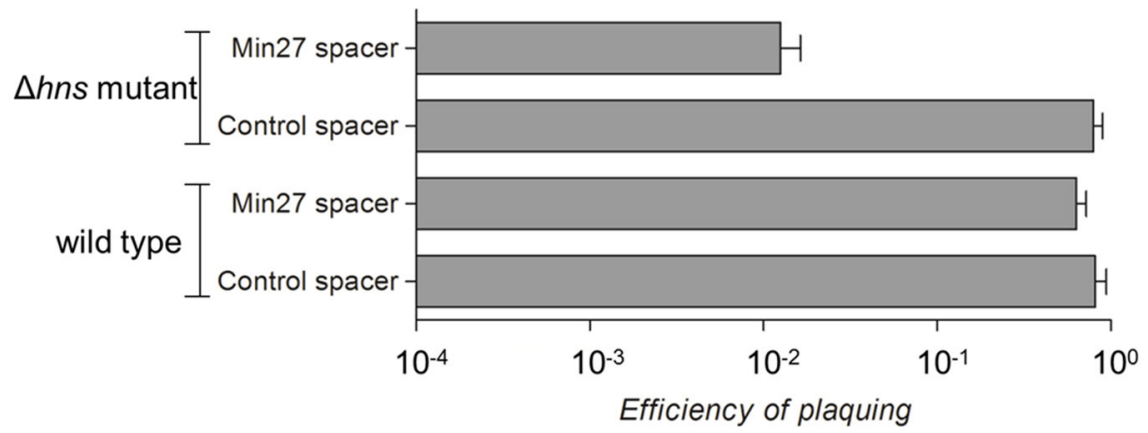

FIGURE 3 | Activated CRISPR-Cas inhibits phage replication. (A) Schematic diagram of the engineered CRISPRs. The sequence of the anti-Min27 spacers is taken from the genome of the Stx phage Min27. Repeats are the same as the CRISPR loci in MG1655. The spacers are homologous protospacers (32 bp) in the phage Min27 genes $O, P, Q$, and $R$, respectively. (B) Four strains, FQ1, FQ2, FQ3, and FQ4, were used to assess their ability to hamper phage propagation. (C) The effect of the CRISPR-Cas system on phage propagation in the four strains was measure by efficiency of plating $(E O P)$ assay. (EOP = phage titer on test

bacterium/phage titer on indicator bacterium MC1061). All assays were conducted at least three times.

H-NS mutation-mediated CRISPR-Cas activity was involved in blocking Shiga toxin expression (Figure 6B).

\section{DISCUSSION}

H-NS is an abundant bacterial protein with DNA and RNA binding activity, and it has been found to be involved in the process of gene transcription silencing (Brescia et al., 2004; Dorman, 2004, 2014). Moreover, the effect of the bacterial H-NS protein on gene expression is overwhelmingly negative (Dorman, 2004). In E. coli, H-NS inhibits the transcription of the precursor crRNA by binding to the AT-rich CRISPR leader promoter to repress the immune activity of type I-E CRISPR-Cas against phages and plasmids (Pul et al., 2010; Medina-Aparicio et al., 2011). Pul et al. (2010) reported that cas A and CRISPR expression is repressed by H-NS in E. coli. In this study, we investigated the relationship between the Stx 2 phage lysogen and E. coli CRISPRCas immune activity based on an H-NS-deficient strain. Since we did not obtain an E. coli K-12 $\Delta$ hns mutant strain from the Keio collection, an hns gene in-frame deletion mutant was generated. The transcription levels of the cas gene were upregulated in the H-NS deficient strain in comparison to the wild-type strain (Figure 1B), which is similar to the findings reported in a previous study (Pul et al., 2010). This result indicates that, by relieving the H-NS repression, the E. coli CRISPR-Cas system seems to be activated. Moreover, the results of the transformation efficiency of the foreign plasmids carrying the homologous spacer into both the wild-type and the $\Delta h n s$ mutant strains further confirmed that H-NS deletion mediates CRISPR-Cas activation (Figure 1C). These data provide experimental evidence that the $\Delta h n s$ mutant strain, which we generated, was CRISPRactivated, and it can be used in further CRISPR-Cas associated investigations.

In addition to the function of adaptive immunity, the CRISPRCas system has also been reported to be involved in modulating other processes, such as the genetic regulation of group behavior and virulence, DNA repairing, and genome evolution (Westra et al., 2014). It is important to note that Zegans et al. (2009) observed that lysogenic infection of Pseudomonas aeruginosa 


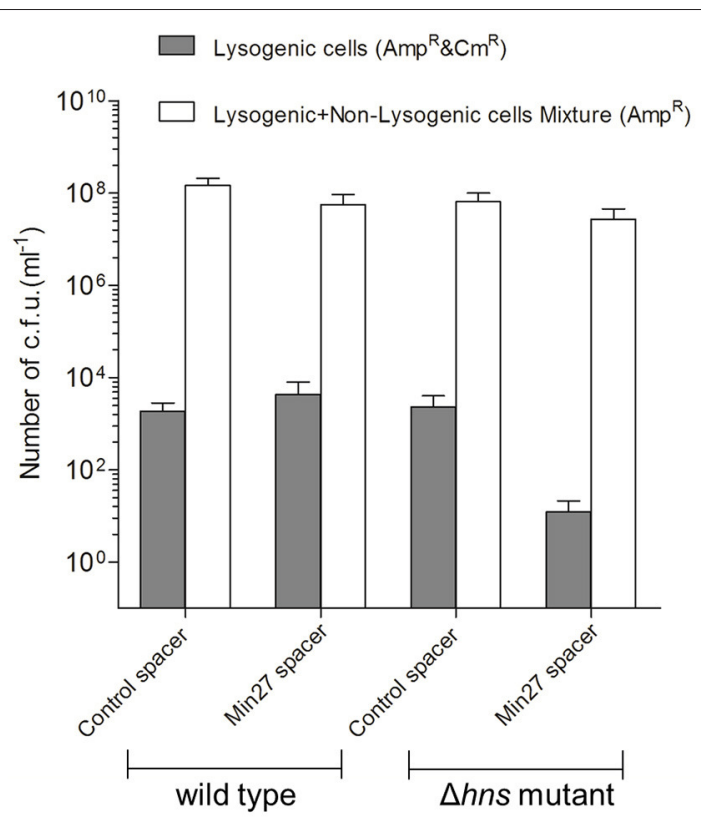

FIGURE 4 | Activated CRISPR-Cas represses the Stx phage lysogenic infection. The recombinant mutant phage Min27( $\Delta$ stx::cat) was used to lysogenically infect wild-type and $\Delta h n s$ mutant MG1655 strains harboring the engineered CRISPRs (containing either the control spacer or the Min27 phage-derived spacer) plasmids (FQ1, FQ2, FQ3, and FQ4). A total of $100 \mu \mathrm{l}$ of filtered phage Min27( $\Delta s t x:$ :cat) was mixed with $100 \mu \mathrm{l}$ of the cultures of the four indicated strains, and the mixtures were incubated overnight at $37^{\circ} \mathrm{C}$. These bacterium-phage mixtures were then plated onto $\mathrm{Amp}^{R}$ (lysogenic and non-lysogenic cells) or $\mathrm{Amp}^{\mathrm{R}}$ plus $\mathrm{Cm}^{\mathrm{R}}$ (lysogens) plates to obtain individual colonies and to calculate the CFU count.

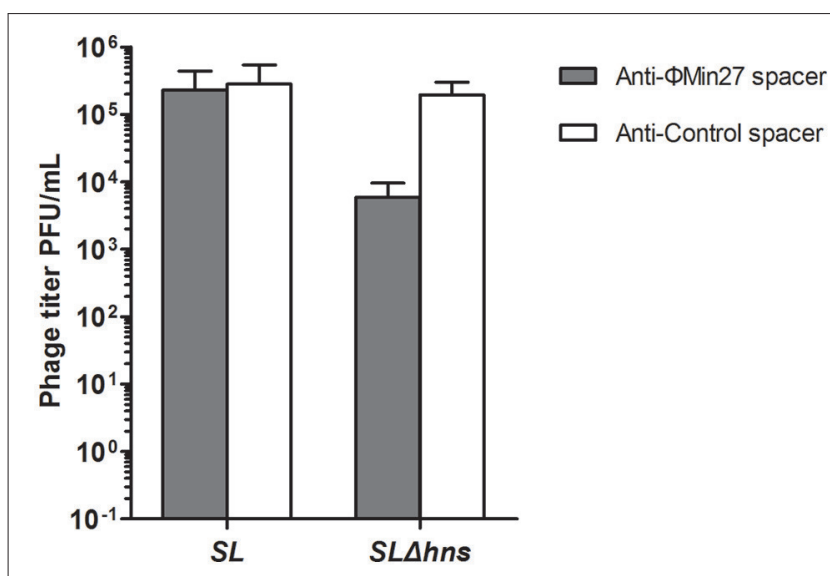

FIGURE 5 | Phage release of the lysogens was determined by PFU assay. Mitomycin $\mathrm{C}$ was added to the culture of lysogens to induce phage release. SL indicates the Stx phage Min27 single lysogen; SL $\Delta h n s$, Stx phage Min27 single lysogen with hns deletion.

PA14 with bacteriophage DMS3 inhibits biofilm formation and swarming motility, both of which are important bacterial group behaviors. This suggests that CRISPR-Cas plays a role in modifying the effects of lysogeny on Pseudomonas aeruginosa
(Zegans et al., 2009). Interestingly, the biofilm formation and swarming motility ability of the Stx2 phage lysogenic $\Delta h n s$ mutant strains were significantly decreased in comparison to the wild-type lysogen. Although additional investigation is necessary, it is possible that the type I-E CRISPR-Cas system may exert some influence on the regulation of group behavior in Stx2 phage lysogens.

In E. coli, and many other types of bacteria, a specialized ribonucleoprotein complex, known as the Cascade, confers immunity by an interference reaction between the CRISPRtranscript RNA (crRNA) and the invader DNA sequences, known as protospacers. The Cascade recognizes the invader DNA via short protospacer adjacent motif (PAM) sequences and crRNA-DNA complementarity (Majsec et al., 2016). Given that the CRISPR system acts against the prophage and the CRISPR system was activated when the hns was deleted, it could protect lysogens from prophage induction and Shiga toxin production. In this study, according to the spacer matching requirements of the CRISPR-Cas interference process, based on the PAM sequence, four protospacers were selected from the phage Min27 genome to construct an engineered CRISPR plasmid. The results show that, in the single lysogen of Min27 phage, which was harboring the phage genome-derived spacers, both progeny phage release and Shiga toxin production were inhibited with the hns deletion in comparison to the control lysogen. It has also been shown that the lysogenic infection efficiency of recombinant mutant phage $\operatorname{Min} 27(\Delta$ stx::cat) against FQ4 ( $\Delta h n s$ mutant harboring Min27 phage spacers) is much lower than it is in FQ1, FQ2, and FQ3 (other three stains). A previous study reported that even low CRISPR activity is still toxic to lysogenic cells carrying targeted spacers (Edgar and Qimron, 2010). Therefore the reduction of phage release and Shiga toxin production might result from CRISPR-activitymeditated bacterial cell death. The bacterial death may be a result of the targeting and neutralization of prophage DNA, leading to bacterial DNA destruction by CRISPR-Cas activity (Edgar and Qimron, 2010). In fact, the survival rate of the lysogens harboring the engineered CRISPR plasmids after mitomycin C induction was detected in our study. It was shown that the survival rate of FQ8 was much lower than the survival rate of FQ7 (data not shown). Although it is possible that the CRISPR system may affect the level of the CI repressor in the cell, which leads to the induction of the prophage, this possibility was ruled out experimentally (Edgar and Qimron, 2010). Another possible explanation is that, in the case of Stx phage, the induction of Stx prophages has been shown to be controlled by the $\operatorname{Rec} A$-a regulator of the SOS bacterial response (Kruger and Lucchesi, 2015; Sheng et al., 2016). It has been shown that the interaction of the Type I-F CRISPR system with the phage protospacer induces expression of the SOS-regulated phage-related genes through the activity of the nuclease Cas3 and subsequent $\operatorname{Rec} A$ activation (Heussler et al., 2015). In this present study, the H-NS-mediated CRISPR-Cas activity might also affect the $\operatorname{Rec} A$ gene expression in order to regulate Stx prophage induction and Shiga toxin production. A previous study indicated that since bacteria can use quorum sensing to regulate their antiphage activities, they could specifically upregulate their defense mechanisms to avoid 


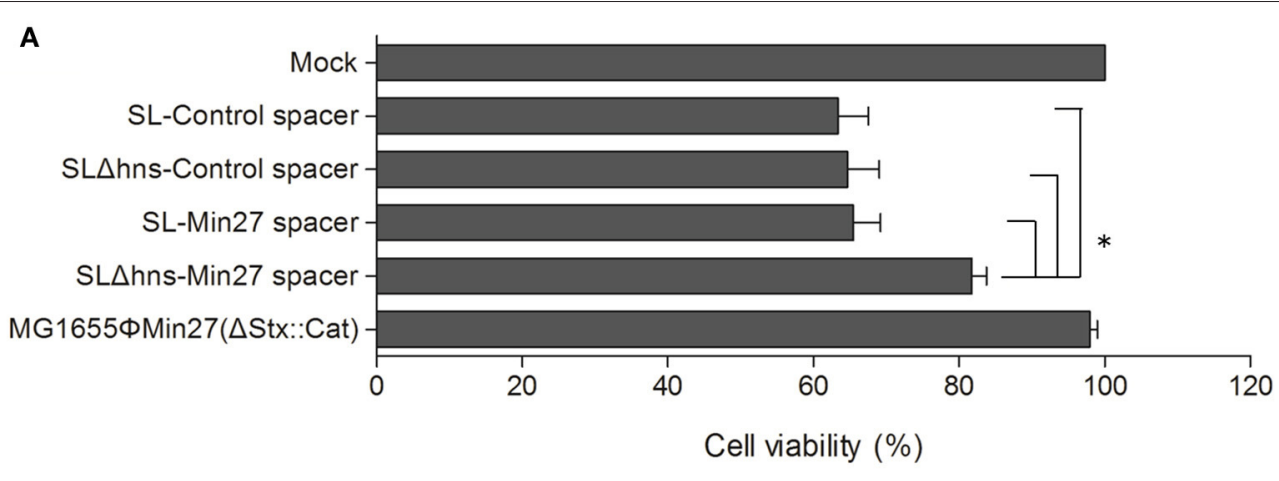

B

ФMin27 Single Lysogen

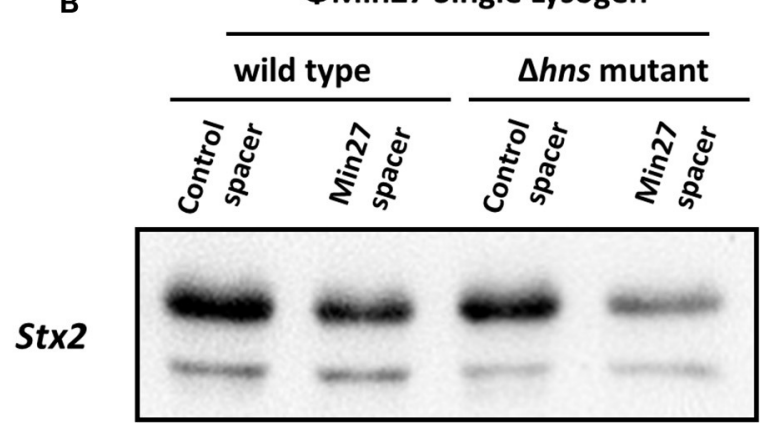

FIGURE 6 | Shiga toxin production of the lysogen was measured using a Vero cell viability assay and Western blot analysis. (A) Serial 10 -fold dilutions of the filtered supernatants were added to the cell monolayer ( $100 \mu \mathrm{l} /$ well) and incubated for $48 \mathrm{~h}$ at $37^{\circ} \mathrm{C}$ under $5 \% \mathrm{CO}_{2}$. The viability of the Vero cells was determined by crystal violet staining. Lysogen, MG1655ФMin27( $\Delta$ stx::cat), which has an inconspicuous cytotoxic effect on Vero cells, was used as a negative control. One-way analysis of variance (ANOVA) was used for data analysis $\left(p<0.05\right.$ is considered to be statistically significant) $\left({ }^{*} p<0.05\right)$. (B) The filtered supernatants were collected and then subjected to saturated ammonium sulfate precipitation. The prepared samples were subjected to SDS-polyacrylamide gel electrophoresis (PAGE), and then examined by Western blot analysis using Stx2 monoclonal antibody (MAb) $11 \mathrm{E} 10$.

infection (Høyland-Kroghsbo et al., 2013). Another possibility is that quorum sensing played an essential role in regulating the type I (type I-E/I-F) CRISPR-Cas system, which may affect phage lysogen biological characteristics (Patterson et al., 2016; HøylandKroghsbo et al., 2017). Further study is needed to determine the mechanism by which intermediates regulate the CRISPRCas system on Shiga toxin production, and to verify if quorum sensing participates in controlling CRISPR adaptive immunity to Stx phage lysogen.

\section{AUTHOR CONTRIBUTIONS}

JS, YY, and QF conceived and designed the experiments; QF and SL performed the experiments; QF, ZW, JM, and YC analyzed the data; QF, YY, WS, and HW

\section{REFERENCES}

Allison, H. E., Sergeant, M. J., James, C. E., Saunders, J. R., Smith, D. L., Sharp, R. J., et al. (2003). Immunity profiles of wild-type and recombinant shiga-like toxin-encoding bacteriophages and characterization of novel double lysogens. Infect. Immun. 71, 3409-3418. doi: 10.1128/IAI.71.6.3409-3418.2003 contributed reagents and materials; QF and JS wrote the manuscript.

\section{ACKNOWLEDGMENTS}

We are indebted to Dr. Daxin Peng of Yangzhou University for providing the lambda recombination system. This research was supported by grants from the National Natural Science Foundation of China (31272580, 31372500, 31672524, 31571932).

\section{SUPPLEMENTARY MATERIAL}

The Supplementary Material for this article can be found online at: http://journal.frontiersin.org/article/10.3389/fmicb. 2017.00652/full\#supplementary-material

Barrangou, R. (2015). The roles of CRISPR-Cas systems in adaptive immunity and beyond. Curr. Opin. Immunol. 32, 36-41. doi: 10.1016/j.coi.2014. 12.008

Barrangou, R., and Marraffini, L. A. (2014). CRISPR-Cas systems: prokaryotes upgrade to adaptive immunity. Mol. Cell 54, 234-244. doi: 10.1016/j.molcel.2014.03.011 
Bloch, V., Yang, Y., Margeat, E., Chavanieu, A., Augé, M. T., Robert, B., et al. (2003). The H-NS dimerization domain defines a new fold contributing to DNA recognition. Nat. Struct. Biol. 10, 212-218. doi: 10.1038/nsb904

Brescia, C. C., Kaw, M. K., and Sledjeski, D. D. (2004). The DNA binding protein $\mathrm{H}-\mathrm{NS}$ binds to and alters the stability of RNA in vitro and in vivo. J. Mol. Biol. 339, 505-514. doi: 10.1016/j.jmb.2004.03.067

Croxen, M. A., Law, R. J., Scholz, R., Keeney, K. M., Wlodarska, M., and Finlay, B. B. (2013). Recent advances in understanding enteric pathogenic Escherichia coli. Clin. Microbiol. Rev. 26, 822-880. doi: 10.1128/CMR.00022-13

Datsenko, K. A., and Wanner, B. L. (2000). One-step inactivation of chromosomal genes in Escherichia coli K-12 using PCR products. Proc. Natl. Acad. Sci. U.S.A. 97, 6640-6645. doi: 10.1073/pnas.120163297

Dorman, C. J. (2004). H-NS: a universal regulator for a dynamic genome. Nat. Rev. Microbiol. 2, 391-400. doi: 10.1038/nrmicro883

Dorman, C. J. (2014). H-NS-like nucleoid-associated proteins, mobile genetic elements and horizontal gene transfer in bacteria. Plasmid 75, 1-11. doi: 10.1016/j.plasmid.2014.06.004

Edgar, R., and Qimron, U. (2010). The Escherichia coli CRISPR system protects from lambda lysogenization, lysogens, and prophage induction. J. Bacteriol. 192, 6291-6294. doi: 10.1128/JB.00644-10

Fu, Q., Sun, J., and Yan, Y. (2015). [The functional aspects of bacterial CRISPR-cas systems and interactions between phages and its bacterial hosts-a review]. Wei Sheng Wu Xue Bao 55, 251-257. doi: 10.13343/j.cnki.wsxb.20140353

Fu, Q., Su, Z., Cheng, Y., Wang, Z., Li, S., Wang, H., et al. (2017). Clustered, regularly interspaced short palindromic repeat (CRISPR) diversity and virulence factor distribution in avian Escherichia coli. Res. Microbiol. 168, 147-156. doi: 10.1016/j.resmic.2016.10.002

Garneau, J. E., Dupuis, M. Ė., Villion, M., Romero, D. A., Barrangou, R., Boyaval, P., et al. (2010). The CRISPR/Cas bacterial immune system cleaves bacteriophage and plasmid DNA. Nature 468, 67-71. doi: 10.1038/nature09523

Heussler, G. E., Cady, K. C., Koeppen, K., Bhuju, S., Stanton, B. A., and O’Toole, G. A. (2015). Clustered regularly interspaced short palindromic repeat-dependent, biofilm-specific death of Pseudomonas aeruginosa mediated by increased expression of phage-related genes. mBio 6, e00129-15. doi: $10.1128 / \mathrm{mBio} .00129-15$

Høyland-Kroghsbo, N. M., Maerkedahl, R. B., and Svenningsen, S. L. (2013). A quorum-sensing-induced bacteriophage defense mechanism. mBio 4, e00362-312. doi: 10.1128/mBio.00362-12

Høyland-Kroghsbo, N. M., Paczkowski, J., Mukherjee, S., Broniewski, J., Westra, E., Bondy-Denomy, J., et al. (2017). Quorum sensing controls the Pseudomonas aeruginosa CRISPR-Cas adaptive immune system. Proc. Natl. Acad. Sci. U.S.A. 114, 131-135. doi: 10.1073/pnas.1617415113

Kleanthous, H., Smith, H. R., Scotland, S. M., Gross, R. J., Rowe, B., Taylor, C. M., et al. (1990). Haemolytic uraemic syndromes in the British Isles, 1985-8: association with verocytotoxin producing Escherichia coli. Part 2: Microbiological aspects. Arch. Dis. Child 65, 722-727. doi: 10.1136/adc.65.7.722

Krüger, A., and Lucchesi, P. M. (2015). Shiga toxins and stx phages: highly diverse entities. Microbiology 161(Pt 3), 451-462. doi: 10.1099/mic.0.000003

Labrie, S. J., Samson, J. E., and Moineau, S. (2010). Bacteriophage resistance mechanisms. Nat. Rev. Microbiol. 8, 317-327. doi: 10.1038/nrmicro2315

Liu, F., Barrangou, R., Gerner-Smidt, P., Ribot, E. M., Knabel, S. J., and Dudley, E. G. (2011). Novel virulence gene and clustered regularly interspaced short palindromic repeat (CRISPR) multilocus sequence typing scheme for subtyping of the major serovars of Salmonella enterica subsp. enterica. Appl Environ. Microbiol. 77, 1946-1956. doi: 10.1128/AEM.02625-10

Louwen, R., Staals, R. H., Endtz, H. P., van Baarlen, P., and van der Oost, J. (2014). The role of CRISPR-Cas systems in virulence of pathogenic bacteria. Microbiol. Mol. Biol. Rev. 78, 74-88. doi: 10.1128/MMBR.00039-13

Majsec, K., Bolt, E. L., and Ivancic-Bace, I. (2016). Cas3 is a limiting factor for CRISPR-Cas immunity in Escherichia coli cells lacking H-NS. BMC Microbiol. 16:28. doi: 10.1186/s12866-016-0643-5

Medina-Aparicio, L., Rebollar-Flores, J. E., Gallego-Hernández, A. L., Vázquez, A., Olvera, L., Gutiérrez-Ríos, R. M., et al. (2011). The CRISPR/Cas immune system is an operon regulated by LeuO, H-NS, and leucine-responsive regulatory protein in Salmonella enterica serovar Typhi. J. Bacteriol. 193, 2396-2407. doi: 10.1128/JB.01480-10

Pacheco, A. R., and Sperandio, V. (2012). Shiga toxin in enterohemorrhagic E. coli: regulation and novel anti-virulence strategies. Front. Cell. Infect. Microbiol. 2:81. doi: $10.3389 /$ fcimb.2012.00081
Patterson, A. G., Jackson, S. A., Taylor, C., Evans, G. B., Salmond, G. P., Przybilski, R., et al. (2016). Quorum Sensing Controls Adaptive Immunity through the Regulation of Multiple CRISPR-Cas Systems. Mol. Cell 64, 1102-1108. doi: 10.1016/j.molcel.2016.11.012

Pul, U., Wurm, R., Arslan, Z., Geissen, R., Hofmann, N., and Wagner, R. (2010). Identification and characterization of E. coli CRISPR-cas promoters and their silencing by H-NS. Mol. Microbiol. 75, 1495-1512. doi: 10.1111/j.1365-2958.2010.07073.x

Schembri, M. A., and Klemm, P. (2001). Biofilm formation in a hydrodynamic environment by novel fimh variants and ramifications for virulence. Infect. Immun. 69, 1322-1328. doi: 10.1128/IAI.69.3.1322-1328.2001

Sheng, L., Rasco, B., and Zhu, M. J. (2016). Cinnamon oil inhibits shiga toxin type 2 phage induction and shiga toxin type 2 production in Escherichia coli O157:H7. Appl. Environ. Microbiol. 82, 6531-6540. doi: 10.1128/AEM.01 702-16

Skyberg, J. A., Siek, K. E., Doetkott, C., and Nolan, L. K. (2007). Biofilm formation by avian Escherichia coli in relation to media, source and phylogeny. J. Appl. Microbiol. 102, 548-554. doi: 10.1111/j.1365-2672.2006.03076.x

Smith, M. J., Melton-Celsa, A. R., Sinclair, J. F., Carvalho, H. M., Robinson, C. M., and O'Brien, A. D. (2009). Monoclonal antibody 11E10, which neutralizes shiga toxin type 2 (Stx2), recognizes three regions on the Stx2 A subunit, blocks the enzymatic action of the toxin in vitro, and alters the overall cellular distribution of the toxin. Infect. Immun. 77, 2730-2740. doi: 10.1128/IAI.00 005-09

Su, L. K., Lu, C. P., Wang, Y., Cao, D. M., Sun, J. H., and Yan, Y. X. (2010). [Lysogenic infection of a Shiga toxin 2-converting bacteriophage changes host gene expression, enhances host acid resistance and motility]. Mol. Biol. (Mosk). 44, 60-73. doi: 10.1134/S0026893310010085

Toutain, C. M., Zegans, M. E., and O’Toole, G. A. (2005). Evidence for two flagellar stators and their role in the motility of Pseudomonas aeruginosa. J. Bacteriol. 187, 771-777. doi: 10.1128/JB.187.2.771-777.2005

van der Oost, J., Jore, M. M., Westra, E. R., Lundgren, M., and Brouns, S. J. (2009). CRISPR-based adaptive and heritable immunity in prokaryotes. Trends Biochem. Sci. 34, 401-407. doi: 10.1016/j.tibs.2009.05.002

Wang, S., Niu, C., Shi, Z., Xia, Y., Yaqoob, M., Dai, J., et al. (2011). Effects of ibeA deletion on virulence and biofilm formation of avian pathogenic Escherichia coli. Infect. Immun. 79, 279-287. doi: 10.1128/IAI.00821-10

Westra, E. R., Buckling, A., and Fineran, P. C. (2014). CRISPR-Cas systems: beyond adaptive immunity. Nat. Rev. Microbiol. 12, 317-326. doi: 10.1038/nrmicro 3241

Westra, E. R., Pul, U., Heidrich, N., Jore, M. M., Lundgren, M., Stratmann, T., et al. (2010). H-NS-mediated repression of CRISPR-based immunity in Escherichia coli K12 can be relieved by the transcription activator LeuO. Mol. Microbiol. 77, 1380-1393. doi: 10.1111/j.1365-2958.2010.07315.x

Westra, E. R., van Erp, P. B., Künne, T., Wong, S. P., Staals, R. H., Seegers, C. L., et al. (2012). CRISPR immunity relies on the consecutive binding and degradation of negatively supercoiled invader DNA by Cascade and Cas3. Mol. Cell 46, 595-605. doi: 10.1016/j.molcel.2012.03.018

Yin, S., Jensen, M. A., Bai, J., Debroy, C., Barrangou, R., and Dudley, E. G. (2013). The evolutionary divergence of Shiga toxin-producing Escherichia coli is reflected in clustered regularly interspaced short palindromic repeat (CRISPR) spacer composition. Appl. Environ. Microbiol. 79, 5710-5720. doi: 10.1128/AEM.00950-13

Zegans, M. E., Wagner, J. C., Cady, K. C., Murphy, D. M., Hammond, J. H., and O’Toole, G. A. (2009). Interaction between bacteriophage DMS3 and host CRISPR region inhibits group behaviors of Pseudomonas aeruginosa. J. Bacteriol. 191, 210-219. doi: 10.1128/JB.00797-08

Conflict of Interest Statement: The authors declare that the research was conducted in the absence of any commercial or financial relationships that could be construed as a potential conflict of interest.

Copyright (c) $2017 \mathrm{Fu}$, Li, Wang, Shan, Ma, Cheng, Wang, Yan and Sun. This is an open-access article distributed under the terms of the Creative Commons Attribution License (CC BY). The use, distribution or reproduction in other forums is permitted, provided the original author(s) or licensor are credited and that the original publication in this journal is cited, in accordance with accepted academic practice. No use, distribution or reproduction is permitted which does not comply with these terms. 